\title{
DIGITAL WATERMARKING AUTHENTICATION AND RESTORATION FOR CHINESE CALLIGRAPHY IMAGES
}

\author{
Anthony T. S. Ho, Xunzhan Zhu and Lilian H. Tang \\ Department of Computing \\ University of Surrey \\ GU2 7XH UK \\ Correspondence Email: a.ho@surrey.ac.uk
}

\begin{abstract}
In this paper, a semi-fragile watermarking method for authentication and automatic restoration of Chinese calligraphy images is proposed using the pinned sine transform (PST). The watermarking system can locate the content of image that has been tampered maliciously with high accuracy. In particular, the watermarking scheme is very sensitive to any texture alteration in the watermarked images, which is crucial for calligraphy image analysis. The tampered portions could be approximately restored. Results of simulation experiments are presented to demonstrate the effectiveness of the proposed method.
\end{abstract}

Index Terms-Security, Image processing, Image restoration

\section{INTRODUCTION}

In the history of art, the authentication of paintings, drawings and many other forms of art are typically performed by human experts. With digital technology available, more and more research activities have been focused on automatic authentication/identification of digitized art works so that not just a more non-destructive in-situ analysis can be performed but potentially objective measurement of the content of art works can be constructed for recommendation, artistic transformation and analysis. Various traditional pattern recognition approaches involving feature extraction and classification processes were applied in such research. One example is Kroner's work [1], which tried to establish the relationship between a drawing and a certain artist. It worked well for free hand drawings and sketches. Similar method was used for identifying the author of fine art paintings based on images content [2]. The work used a wavelet-based technique to measure the stroke texture and regarded such information as a feature to describe the strokes in the painting. Classification was performed through a fuzzy K-NN algorithm. Lyu et al [3] described a computational technique for authenticating paintings and drawings. The approach built a statistical model of an artist from the images of a set of authenticated works and against which new works were compared.

Digital watermarking techniques have recently attracted much attention in many applications but little work has been carried out in art domain. Zhao et al [4] developed digital watermarking and data hiding framework coupled with image processing techniques for compression and preservation of integrity of culture heritage imagery. Semifragile watermarking was applied in this work. With the increasing need of heritage preservation and artistic presentation in commercial and domestic use, Chinese calligraphy works become more and more available in digital formats. Digitized Chinese calligraphy, like many other forms of art, is vulnerable to malicious attacks. Tsai et al [5] proposed a method that tried to distinguish authenticated processing and malicious manipulation using a character-based authentication scheme. In the scheme the edge information of Chinese characters are extracted to form a feature image where the authentication message is generated for locating the tampered Chinese characters.

In this paper, we propose a novel semi-fragile watermarking scheme for Chinese calligraphy image authentication using the pinned sine transform (PST), which is based on our previous work [6]. A semi-fragile watermarking is designed to be unaffected by legitimate manipulations, but less robust against by illegitimated distortions. In our scheme, the original image is decomposed into two mutually uncorrelated fields, namely, the boundary field and the pinned field. The texture information of the original image is contained in the pinned field, wherein the sine transform is equivalent to a fast Karhunen-Loeve transform (KLT). By exploiting this important property, we propose to embed a watermark signal into the sine transform domain of the pinned field for content authentication. As illustrated in this paper, the proposed watermarking scheme is especially sensitive to texture alterations of the host image. This provides significant advantage for authentication of calligraphy images, of which analysis is strongly texture based. Moreover, although our scheme is block-wise, the watermarking of one block is closely related to all the 
blocks surrounding it, which renders our scheme robust to the "cutting and pasting" attacks. The next section presents a brief review of the PST. The watermark embedding and authentication methods are elaborated in Section 3 and Section 4, respectively. Section 5 gives the simulation results followed by the conclusion in Section 6 .

\section{PINNED SINE TRANSFORM}

Suppose a data vector

$$
\mathbf{X}=\left[x_{0} \ldots x_{n+1}\right]^{T}
$$

is separated into a boundary response $\mathbf{X}^{b}$ defined by $x_{0}$ and

$x_{n+1}$, and a residual sequence $\mathbf{X}^{\prime}-\mathbf{X}^{b}$, where

$$
\mathbf{X}^{\prime}=\left[x_{1} \ldots x_{n}\right]^{T} .
$$

In [7], Jain showed that if $\mathbf{X}$ was a first-order stationary Gauss-Markov sequence, the sequence $\mathbf{X}^{\prime}-\mathbf{X}^{b}$ had the sine transform as its KLT.

Extending the above theory to the more general 2-D case, Meiri et al $[8,9]$ proposed the decomposition of an image field into two sub-fields, namely, the boundary field and a residual field. The boundary field depends only on the block boundaries and for the residual field, so-called the pinned field in [9], which vanishes at the boundaries, its KLT is the sine transform.

The semi-fragile watermarking adapts to a selective authentication scheme on the content of images. The primary textures of the Chinese calligraphy images, such as edges, are of special interest to Chinese calligraphy image analysis, for example, to determine the shapes of the characters. Therefore, our scheme aims at protecting the primary textures of the Chinese calligraphy images. To this end, the watermark should not survive the authentication process if such textures are tampered or damaged. The results of the decomposition of a Chinese calligraphy image are shown in Fig. 1. We find that the boundary field is only a blurred version of the original image, whilc the pinned field is a good characterization of edges, which fully reflects the texture information in the original image. Thus the watermark can be embedded into the pinned field as an indicator of the authenticity of the watermarked image. Moreover, since most common image manipulations tend to preserve such primary features of images, this embedding method ensures that the watermark does not suffer significantly from such legitimate manipulations.

In PST, the image is divided into overlapped blocks, which introduces an inter-block relationship to the pinned sine transformed images. Therefore the watermarking of any particular block also depends on its location in the image instead of depending only on its own content. Thus, simple "cutting and pasting" counterfeiting attack can be exposed by this encoding scheme since the counterfeit of one block affects all the blocks around it.

\section{WATERMARK EMBEDDING}

The semi-fragile watermarking aims at authentication of the semantic content of images, i.e., they should protect the primary features of the image content, e.g., edges. The watermark should not survive if such features are damaged. As we have seen in the previous section, after the dualcomponent decomposition, information of prominent edges of the original image is contained in the pinned field. Thus we may embed the watermark into the pinned field as an indication of the authenticity of the watermarked image.

The original image $\mathbf{X}$ is partitioned into overlapping blocks $\left\{\mathbf{X}_{m, n}\right\} \in \mathbf{X}$ of size $10 \times 10$, where $m$ and $n$ are the coordinate numbers of this block. Two neighboring blocks are overlapped by one column or row. For every block, the surrounding zone of a 2-pixel width is averaged to generate the initial boundaries and corners. These parameters are used to achieve the boundary field by interpolation and the pinned field is in turn obtained by subtracting the boundary field from central $8 \times 8$ part of the original block. After every block has been decomposed, it results in nonoverlapping pinned field blocks and boundary field blocks, denoted as $\left\{\mathbf{X}_{m, n}^{p}\right\}$ and $\left\{\mathbf{X}_{m, n}^{b}\right\}$, respectively. Both the boundary field and the pinned field are of size of $8 \times 8$.

The watermarking process proceeds by conducting the sine transform to every $\left\{\mathbf{X}_{m, n}^{p}\right\}$ block and by embedding a pseudo-random binary sequence of length $L$ into each block, whose initial seed is contained in a secret key file. In the middle to high frequency bands of the sine transform coefficients, we select, according to the length of the watermark sequence, $L$ coefficients for watermarking modulation. More specifically, the watermarking process is defined as follow:

$$
y^{p}=\left\{\begin{array}{cc}
x^{p} & \left(w=1 \cap x^{p}>T\right) \\
\alpha_{1} & w=1 \cap x^{p} \leq T \\
\alpha_{2} & w=0 \cap x^{p} \geq-T,
\end{array}\right.
$$

where $x^{p}$ and $y^{p}$ are the coefficients before and after watermarking, respectively. $T$ is a sufficiently large positive threshold value, which is set to achieve the best trade-off between the perceptual quality and robustness. $\alpha_{1}$ and $\alpha_{2}$ are floating point values with $\alpha_{1} \in[T / 2, T]$ and $\alpha_{2} \in[-T,-T / 2]$. The watermarked coefficients are then inverse sine transformed and a watermarked image is obtained by adding the boundary field to the watermarked pinned field. 


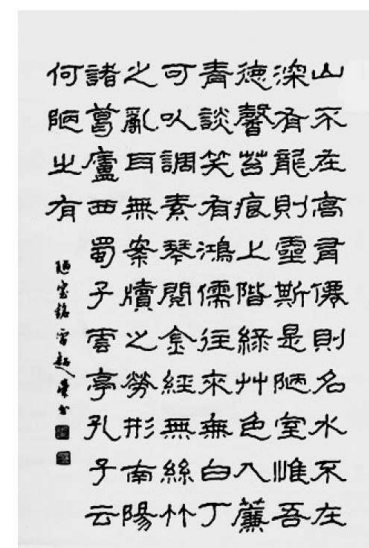

(a)

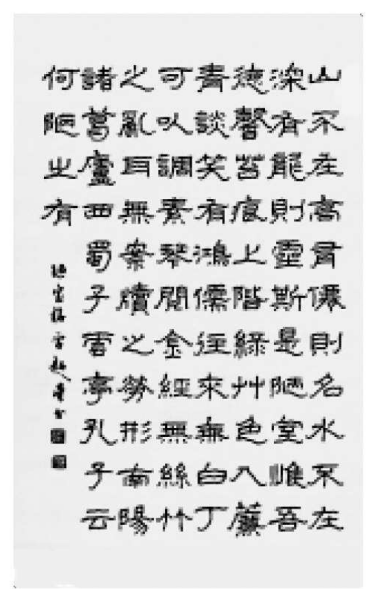

(b)

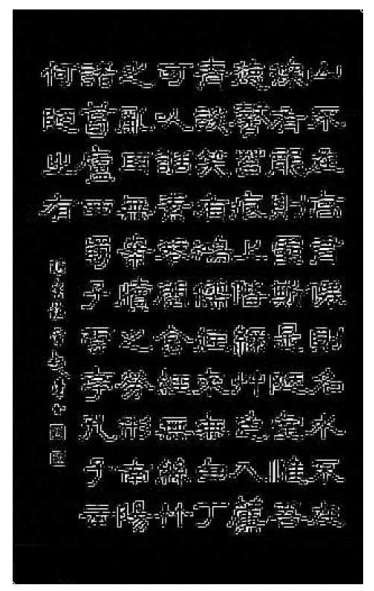

(c)

Fig. 1. The dual-field decomposition: (a) the original imge; (b) the boundary image and (c) the pinned field.

\section{WATERMARK DETECTION AND IMAGE AUTHENTICATION}

The detection of watermark is performed as follows. The detection system receives as input a watermarked and possibly tampered image $\hat{\mathbf{Y}}$. After a similar block-wise decomposition as in the watermark embedding, we obtain the pinned field blocks $\left\{\hat{\mathbf{Y}}_{m, n}^{p}\right\}$. A Sine transform is performed on these blocks. The watermarked coefficients are then located and checked based on the following conditions: if $\hat{y}^{p}>0$, we decide the watermark bit as " 1 "; otherwise, we decide it as " 0 ".

After collecting all the watermark bits in one block, we obtain the retrieved and possibly corrupted watermark. The original watermark is also generated using the initial seed in the key file. The watermark bits are compared via the normalized cross correlation function:

$$
\rho=\frac{\sum_{l=0}^{L} \hat{w}_{m, n}[l] w_{m, n}[l]}{\left[\sum_{l=0}^{L}\left(\hat{w}_{m, n}[l]\right)^{2}\right]^{1 / 2}\left[\sum_{l=0}^{L}\left(w_{m, n}[l]\right)^{2}\right]^{1 / 2}}
$$

Where $w_{m, n}$ is the watermark signal and $\rho \in[-1,1]$. The integrity of the block $\hat{\mathbf{Y}}_{m . n}$ is evaluated according to the value of $\rho$. If no tampering ever occurred to this block, $\rho \rightarrow 1$; on the other hand, $\rho$ will decrease due to different tampering of $\hat{\mathbf{Y}}_{m . n}$. If the content of the block has been changed, i.e. the block has been replaced, due to properties of the normalized cross correlation function, $\rho$ will be extremely low.
Assume $\gamma$ is a properly set threshold, the block is considered to be maliciously tampered if $\rho<\gamma$. The threshold is determined mathematically or experimentally so as to maximize the probability of detection subject to a given probability of false alarm. In our current investigation, $\gamma$ is experimentally set to tolerate unavoidable non-malicious modifications in some practical applications, such as JPEG compression and noise addition, while maintaining the sensitivity of the authentication process to malicious modification on the content of the watermarked images. When tampered portion is detected, the portion can be recovered using the method of projection [10].

\section{SIMULATION RESULTS}

We use the Chinese calligraphy images in BMP format as shown in Fig. 2(a) to test our authentication algorithm, which is of size $512 \times 512$. Fig. 2(b) displays the watermarked image. We can observe that the watermarked image looks identical to the original image, with peak signal to noise ratio (PSNR) value of approximately $40 \mathrm{~dB}$. We then modified the content of the watermarked image: in Fig. 3(a), some characters are exchanged. As illustrated in Fig. 3(b), the maliciously modified areas were accurately detected and identified. The restoration result is also shown in Fig. 3(c). The probability of tamper detection is $\$ 981 \% \$$ and the quality of the recovered portions is $28 \mathrm{~dB}$.

\section{CONCLUSION}


In this paper, we discussed a novel semi-fragile watermarking method using the pinned sine transform for the authentication of Chinese calligraphy images. The watermark was embedded into the pinned field, which contained the texture information of the original image. This

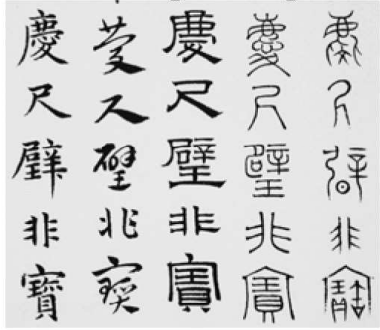

(a) important property of the pinmed field provides the scheme with special sensitivity to any texture alteration to the Chinese calligraphy images. The watermarking system can locate the portions of a watermarked image that have been

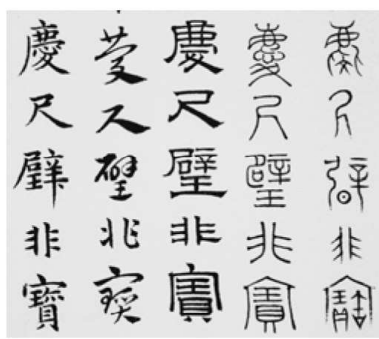

(b)

Fig. 2. Experimental results: (a) The original image; (b) the watermarked image.

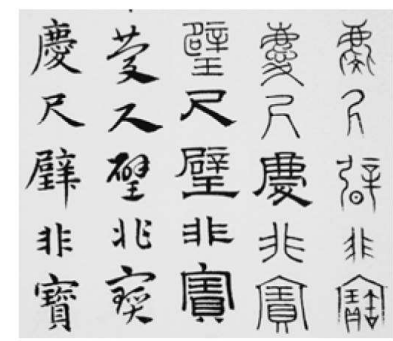

(a)

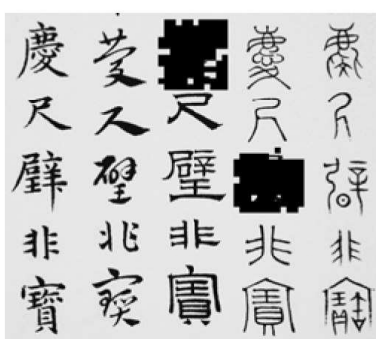

(b)

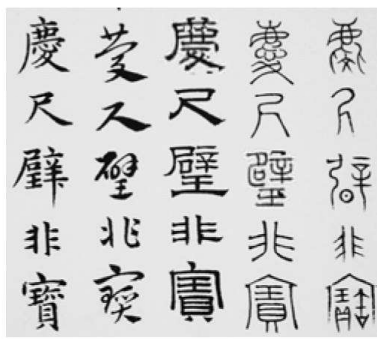

(c)

Fig. 3. Experimental results: (a) the tampered image: (b) the authentication result and (c) the restoration result.

tampered maliciously with high accuracy, and provide the capability for self-correction of the tampered regions.

\section{REFERENCES}

[1] S. Kroner and A. Lattner, "Authentication of free hand drawings by pattern recognition methods," in Proceedings of Fourteenth International Conference on Pattern Recognition, Aug. 1998, pp. 462-464.

[2] G. N. Teixeira, R. Q. Feitosa, and S. Paciornik, "Pattern recognition applied in fine art authentication," in 6th World Multiconference on Systemics, Cybernetics and Informatics, July 2002, pp. 36-39.

[3] S. Lyu, D. Rockmore, and H. Farid, "A digital technique for art authentication," in Proceedings of the National Academy of Sciences of the United States of America, pp. 17006-10.

[4] Y. Zhao, P. Campisi, and D. Kundur, "Dual domain watermarking for authentication and compression of cultural heritage images," IEEE Trans. on Image Processing, pp. 430-448, Mar 2004.
[5] P. Tsai, M.-H. Lin and C.-C. Chang,"A character-based authentication scheme for Chinese calligraphy images," in ICICSPCM 2003, Dec. 2003, pp. 960-964.

[6] A. T. S. Ho, X, Zhu and Y. L. Guan, "Image content authentication using pinned sine transform," EURASIP Journal on Applied Signal Processing, Special Issue on Multimedia Security and Rights Management, vol. 2004, no. 14, pp. 2174-2184, Oct 2004.

[7] A. K. Jain, "Some new techniques in image processing," in Image Science Mathematics, O. Wilde and E. Barrett, Eds. Western Period., California, 1976.

[8] A. Z. Meiri, "The pinned Karhunen-Loeve transform of a two dimensional Gauss-Markov field," in Proc. SPIE Conf. Image Processing, San Diego, CA, 1976.

[9] A. Z. Meiri and E. Yudilevich, "A pinned sine transform image coder," vol. COM-29, pp. 1728-1753, Dec. 1981.

[10] J. Fridrich and M. Goljan, "Images with self-correcting capabilities," in IEEE International Conf. on Image Processing, Kobe, Japan, Oct. 1999, pp. 792-796. 